\title{
Evaluation of different quantization resolution levels on the BER performance of massive MIMO systems under different operating scenarios
}

\author{
Hayder Khaleel AL-Qaysi, Tahreer Mahmood, Khalid Awaad Humood
}

Department of Electronic Engineering, College of Engineering, University of Diyala, Iraq

\begin{tabular}{|c|c|}
\hline Article Info & ABSTRACT \\
\hline Article history: & The massive MIMO system is one of the main technologies in the fifth \\
\hline Received May 1, 2021 & $\begin{array}{l}\text { generation }(5 \mathrm{G}) \text { of telecommunication systems, also recognized as a highly } \\
\text { large-scale system. Constantly in massive MIMO systems, the base station }\end{array}$ \\
\hline Revised Jul 7, 2021 & (BS) is provided with a large number of antennas, and this large number of \\
\hline Accepted Jul 14, 2021 & $\begin{array}{l}\text { antennas need high-quantization resolution levels analog-to-digital } \\
\text { converters (ADCs). In this situation, there will be more power consumption }\end{array}$ \\
\hline Keywords: & suggested method to investigate and analyze the effects of different \\
\hline BER & quantization resolution levels of $\mathrm{ADCs}$ on the bit error rate (BER) \\
\hline Massive MIMO & performance of massive MIMO system under different operating scenarios \\
\hline Quantization resolution levels & $\begin{array}{l}\text { software. The results show that the SNR exceeds } 12 \mathrm{~dB} \\
0.001 \% \text { of BER signals when the number of antennas } 60\end{array}$ \\
\hline ADCs & with low quantization a 2 bits' levels ADCs, approximately. But when the \\
\hline RF chain & antenna number rises to 300 , the SNR exceeds $12 \mathrm{~dB}$ accounts for almost \\
\hline & $\begin{array}{l}0.01 \% \text { of BER transmitted signals. Comparably with the BER performance } \\
\text { of high quantization, } 4 \text { bits-quantization resolution levels ADCs with the } \\
\text { same different antennas have a slight degradation. Therefore, the number of } \\
\text { antennas is a very important influence factor. }\end{array}$ \\
\hline
\end{tabular}

This is an open access article under the $\underline{C C B Y-S A}$ license.

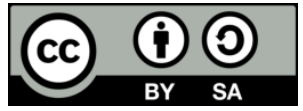

\section{Corresponding Author:}

Hayder Khaleel AL-Qaysi

Department of Electronic Engineering

College of Engineering, University of Diyala

Baqubah, Diyala Province, Iraq

Email: hay.kha.82@uodiyala.edu.iq

\section{INTRODUCTION}

Massive multi-input multi-output (MIMO) is a highly large-scale physical layer system. The Massive MIMO is considered one of the reliable technologies in fifth-generation (5G) telecommunication systems and beyond to improve spectral efficiency, energy efficiency, throughput, security and robustness, hardware complexity, cost, and signal processing [1]-[9]. The main idea in the massive MIMO system is to use a large number of transmitting antennas $\left(T_{X}\right)$ in the base station (BS) than the number of receiving antennas $\left(T_{R}\right)$, which represent the users, to provide simultaneous service within the coverage area with relatively simple signals processing [6], [7], [10]. However, the use of a large number of antennas in the BS of massive MIMO system increases the hardware cost and complexity, and power consumption of the circuit components in the radio frequency (RF) chain that consists of signal mixers, power amplifiers, dedicated filters, and high-quantization resolution level analog-to-digital converters (ADCs) [11], [12]. Consequently, the increased hardware costs and power consumptions represent a substantial obstacle in the practical implementation of the massive MIMO systems [13]. 
The power consumed by high-quantization resolution level ADCs represents the essential part of the total power consumed by the other circuit components of the RF chain [14], [15]. In parallel, the utilization of high-quantization resolution level ADCs contributes significantly to increasing the hardware costs and complexities and reducing quantization errors in the massive MIMO systems [16]-[18]. The power consumption in the ADCs decreases exponentially with quantization resolution levels (quantization bits) and linearly with sampling frequencies (sampling rates) [19]. Particularly, ADCs represent the main parts of receivers in the massive MIMO systems to convert the analog signals into digital signals through sampling and quantization processes and preparing them for subsequent digital signal processing operations [20].

In the literature, many investigations work conducted to study the effect of different quantization resolution levels of ADCs on the performance of massive MIMO systems under different operating scenarios, such as: using a different number of antennas, various modulation schemes, and signal symbols detectors. In [14], the authors investigated the effect of 3-to-12 bits-quantization resolution levels ADCs on the uplink energy efficiency (EE) in the massive MIMO system, with time division duplexing (TDD) mode and a different number of antennas at the BS using linear zero-forcing (ZF) and zero-forcing successive interference cancellation (ZFSIC) signal symbols detectors, with both perfect and imperfect channel state information (CSI). Their results showed that the consumed power is significant in the massive MIMO system when using low (3bits)-quantization resolution levels ADCs due to the increase in quantization errors, which needs to compensate with a large number of antennas at the BS. In addition, the use of the perfect CSI-based linear ZFSIC signal symbols detector is able to improve the EE of the system more than the perfect CSIbased linear ZF signal symbols detector. The authors in [16] investigated the role of using low-quantization resolution levels ADCs with the linear minimum mean squared error (LMMSE) and nonlinear lattice reduction-based successive interference cancellation (LR-SIC) signal symbols detectors in improving the EE in the massive MIMO systems under different operating scenarios. Their results showed that the bit error rate (BER) performance using the LR-SIC signal symbols detectors-based receivers is better than that of LMMSE signal symbols detectors-based receivers and that the EE of the system depends on the number of antennas, cell size, and signal processing efficiency employed at the BS. In addition, the authors suggested that for high EE, signal symbols detectors should be carefully selected and according to an appropriate operating scenario. In order to improve EE, they also suggested using medium (6-to-7 bits)-quantization resolution levels ADCs to achieve a trade-off between power consumed and nonlinear distortion.

Moreover, Shao et al, [21] proposed to increase the uplink EE of the massive MIMO systems under different operating scenarios, based on the use of low (1 bit)-quantization resolution levels ADCs, iterative detection and decoding (IDD) technique, and linear-low resolution aware MMSE (LRA-MMSE) signal symbols detector. Their results yielded a high EE gain and improved BER performance for the massive MIMO system. In [22], the authors investigated the EE in the massive MIMO system using arbitrary (1, 2, and infinity bits)-quantization resolution levels ADCs, superimposed pilots (SP) technology, and LMMSE and maximum ratio combining (MRC) signal symbols detectors under different operating scenarios. Their results showed that the pilots needed to specify a greater power in the massive MIMO system with higher quantization resolution levels ADCs or with a larger number of antennas at the BS. In addition, SP technology is superior to time-multiplexed pilots (TP) technology for low (1 bit)-quantization resolution levels ADCs, and SP technology is more suitable when using higher-quantization resolution levels ADCs and the used number of antennas in the BS is small.

Furthermore, the authors in [23] investigated the effects of using low (1-to-3 bits)-quantization resolution levels ADCs and the number of antennas available at the base station using LMMSE signal symbols detector in improving the uplink spectrum efficiency (SE) of the massive MIMO system under different operating scenarios. Their results proved to improve SE by more than $90 \%$ using low (3 bits)quantization resolution levels ADCs. In addition, the SE improved when using 1 bit-quantization resolution levels ADCs with an increase in the number of antennas at the base station. In [24], the authors proposed a novel hybrid independent component analysis (HICA) signal symbols detector in order to improve the SE and reliability of the MIMO system under different operating scenarios. Their results showed that the HICA signal symbols detector showed improvement in the BER and MSE performances, but it was inefficient and failed to reduce the peak to average power rate (PAPR) rate compared to other state-of-the-art signal symbols detectors such as ICA and wavelet denoising ICA (WDICA).

In [25], the authors investigated the joint effects of the low (1 bit)-quantization resolution levels ADCs and the hardware impairments in the massive MIMO system, using a novel proposed alternating direction method of multipliers (ADMM)-based signal symbols detector, quadrature phase-shift keying (QPSK) modulation, and different numbers of antennas at the BS. The results of their investigations showed that the ADMM-based signal symbols detector achieved better performance than the other state-of-the-art linear signal symbols detectors in terms of improving BER performance and the gain with an increase in the number of antennas and users in the massive MIMO system. 
In this paper, the main objective is to evaluate and measure the influences of different quantization resolution levels of analog-to-digital converters on the BER effectiveness in the massive MIMO system under different operating scenarios using the LMMSE signal symbols detector. The remainder of this paper is arranged: The descriptions of the quantized massive MIMO system equations with the LMMSE signal symbols detector are presented in Section 2. Matlab simulation results that explain the influence of quantization resolution levels analog-to-digital converters on the BER efficiency in the massive multipleinput, multiple-output system are presented in Section 3. Finally, Section 4 concludes this paper.

\section{QUANTIZED MASSIVE MIMO WITH LMMSE SIGNAL SYMBOLS DETECTOR}

First, in this section, we present the quantized massive MIMO system model, where differentquantization resolution levels ADCs are used in the receiver at the BS. Then we present the LMMSE detector to detect the transmitted signal symbols vector by the users at the BS receiver.

\subsection{Quantized massive MIMO model with ADCs}

We consider that the uplink of the massive multiuser (MU) MIMO system, as shown in Figure 1, has a total $\mathrm{M}$ antennas at the $\mathrm{BS}$ and simultaneously serves $\mathrm{K}$ single-antenna users, where $\mathrm{M} \gg \mathrm{K} \gg 1$. Thus, the channel matrix $(\mathrm{H})$ connecting the channel coefficients between $\mathrm{K}$ single-antenna users and $\mathrm{M}$ antennas at the $\mathrm{BS}$ is given by (1) [7], [26], [27],

$$
\mathrm{H}=\left[\begin{array}{ccc}
\mathrm{h}_{11} & \cdots & \mathrm{h}_{1 \mathrm{j}} \\
\vdots & \ddots & \vdots \\
\mathrm{h}_{\mathrm{i} 1} & \cdots & \mathrm{h}_{\mathrm{ij}}
\end{array}\right]
$$

where $\mathrm{H} \in \mathbb{C}^{\mathrm{M} \times \mathrm{K}}$ and the element $\mathrm{h}_{\mathrm{ij}}$ represent the channel attenuation coefficient from the ith receiving antenna to the jth transmitter antenna. The channel matrix $(\mathrm{H})$ in the massive MIMO system provides us with the necessary knowledge about the channel state information (CSI) and thus helps solve many problems that we face in wireless communication systems such as path losses, fading, shadowing, scattering, diffractions, and properties of the channel [27], [28].

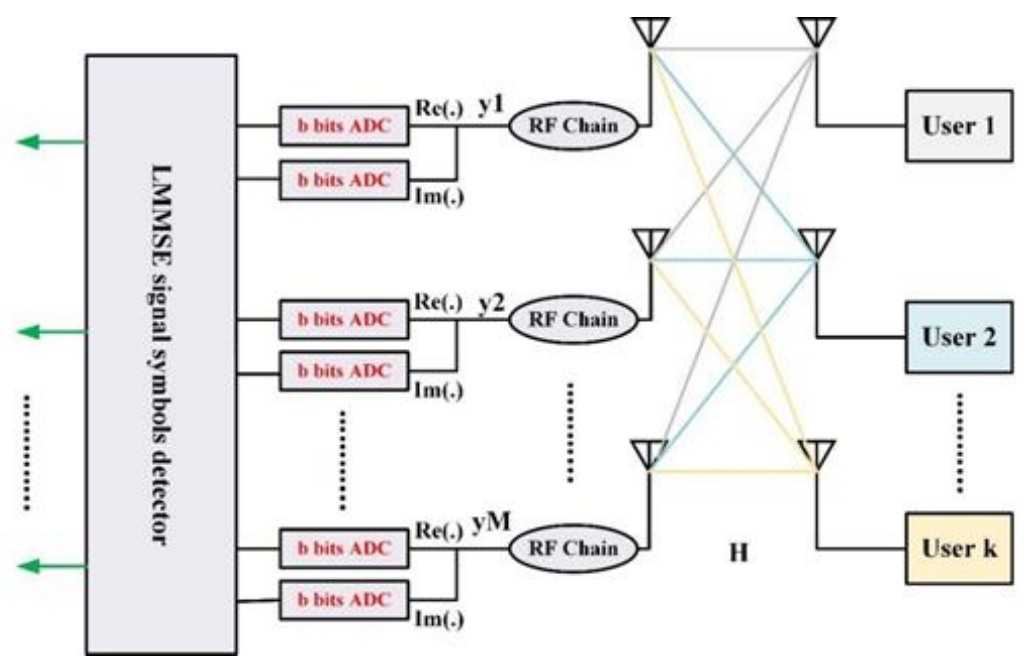

Figure 1. Uplink system model of quantized massive MU-MIMO

The information signals symbols $\left(\mathrm{a}_{\mathrm{K}}\right)$ transmitted by all the $\mathrm{K}$ single-antenna users are encoded first by the channel encoders and then modulated according to the given modulation schemes to the signal symbols $\left(\mathrm{x}_{\mathrm{K}}\right)$. These modulated signal symbols $\left(\mathrm{x}_{\mathrm{K}}\right)$ are transmitted through the channels. The $\mathrm{M}$ antennas at the BS receive these modulated signal symbols $\left(\mathrm{x}_{\mathrm{K}}\right)$ corrupted by the channel effects and noise. The corrupted and unquantized signal symbols vector received in the base station is given by (2),

$$
\mathrm{y}=\mathrm{Hx}+\mathrm{n}
$$


where $\mathrm{x}=\left[\mathrm{x}_{1}, \mathrm{x}_{2}, \mathrm{x}_{3}, \ldots \ldots, \mathrm{x}_{\mathrm{K}}\right]^{\mathrm{T}}$ is the vector of the transmitted signal symbols from all users, $\mathrm{y}=$ $\left[\mathrm{y}_{1}, \mathrm{y}_{2}, \mathrm{y}_{3}, \ldots \ldots . \mathrm{y}_{\mathrm{M}}\right]^{\mathrm{T}}$ is the vector of the received signal symbols, and $\mathrm{n}$ is the additive white Gaussian nose vector. The model given in (2) represents the massive MIMO system model through which it is possible to find the channel matrix $(\mathrm{H})$ based on the provided information about the $\mathrm{x}$ and $\mathrm{y}$ signal symbols vectors.

Generally, the structure of ADCs consists of filters, sampling and hold circuits, and quantizers. Particularly, the quantizers represent the cores of ADCs that perform the quantization processes for the received signal symbols vector (y) given in (2) into the quantized signal symbols version vector (r) [14], [18]. As shown in Figure 1, by utilization of two b bit-quantization resolution levels ADCs for the real and imaginary parts of the complex received signal symbols vector (y), the outcome quantized signal symbols vector (r) is given by (3),

$$
\mathrm{r}=\mathcal{Q}(\mathrm{y})=\mathcal{Q}\left[\mathcal{R}_{\mathrm{e}}(\mathrm{y})+\mathrm{j} \mathrm{I}_{\mathrm{m}}(\mathrm{y})\right]
$$

where $\mathcal{Q}$ (.) describes the quantization process for the real $\left(\mathcal{R}_{\mathrm{e}}(\mathrm{y})\right)$ and imaginary $\left(\mathrm{I}_{\mathrm{m}}(\mathrm{y})\right)$ parts of the complex received signal symbols vector $(\mathrm{y})$, and it is a non-linear process.

The characteristics of 3 bits regular mid ascending quantization processes are shown in Figure 2. Assuming that $\mathcal{Q}$ (.) representing b bits regular mid ascending quantization processes achieved by the quantizer with $\mathrm{N}=2^{\mathrm{b}}$ resolution levels of the ADCs. Consequently, the quantizer performs the conversion process to the real part of the input signal symbols vector (y) into a real evaluated output signal symbols vector $(r)$, where $r=\left[r_{1}, r_{2}, r_{3}, \ldots \ldots, r_{s}\right]$ and $s=1,2,3, \ldots, N$. The endpoints of the input interval $\left(y_{s}\right)$ are given by (4),

$$
y_{s}=\left\{\begin{array}{cl}
-\infty & \text { for } s=1 \\
(-N / 2-1+s) \Delta & \text { for } s=2,3,4, \ldots \ldots, N \\
+\infty & \text { for } s=N+1
\end{array}\right.
$$

where $\Delta$ represents the period size of quantization process. The produced signal symbols values $\left(\mathrm{r}_{\mathrm{s}}\right)$ of the quantizer are given by (5),

$$
\mathrm{r}_{\mathrm{s}}=\left(-\frac{\mathrm{N}}{2}-\frac{1}{2}+\mathrm{s}\right) \Delta \quad \text { for } \mathrm{s}=2,3,4, \ldots \ldots, \mathrm{N}
$$

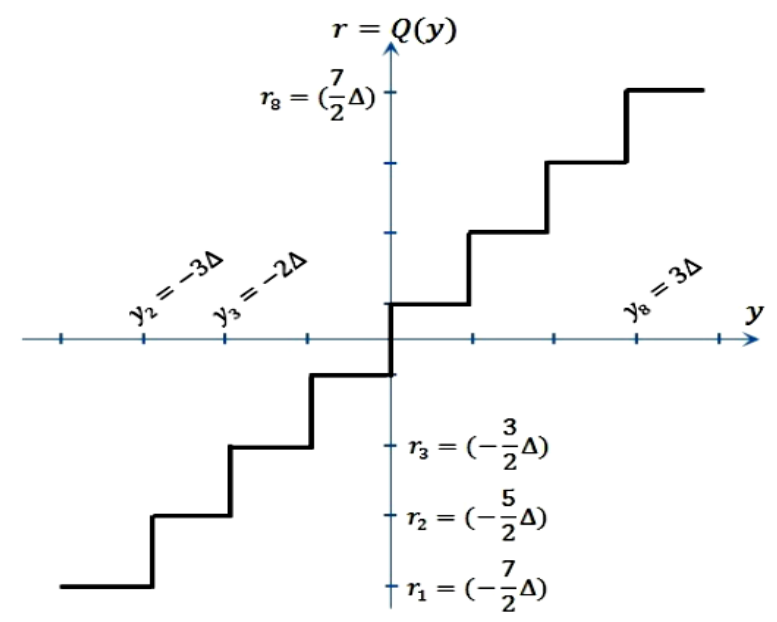

Figure 2. Staircase representation of a three bits regular mid ascending quantization processes

\subsection{LMMSE signal symbols detector}

As a result of the interuser interference among the users, the signal symbols detector is used to evaluate the vector of the transmitted signal symbols (x) from the vector of the received signal symbols (y). In this work, we have adopted the LMMSE signal symbols detector from the other state-of-the-art detectors because of its advantages, including [7], [16],

- It has lower computational complexity.

- It has noise improvement.

- $\quad$ It outperforms the ZF signal symbol detector. 
Implementation the linear signal detector by using $\mathrm{M} \times \mathrm{K}$ matrix (A) to the received signal symbols vector $(\mathrm{y})$, the transmitted signal symbols vector is given by $(6)$,

$$
\hat{x}=A^{H} y=A^{H}(H x+n)
$$

where $\hat{\mathrm{x}}=\left[\hat{\mathrm{x}}_{1}, \hat{\mathrm{x}}_{2}, \hat{\mathrm{x}}_{3}, \ldots \ldots, \hat{\mathrm{x}}_{\mathrm{K}}\right]^{\mathrm{T}}$ is $\mathrm{K} \times 1$ signal characters vector consists of data information stream of $\mathrm{K}$ single-antena user. Employing the LMMSE signal symbols detector by defining the detection matrix data stream (A) as given by (7),

$$
\mathrm{A}=\mathrm{H}\left[\mathrm{H}^{H} H+\frac{\sigma_{\mathrm{n}}^{2}}{\sigma_{\mathrm{x}}{ }^{2}} \mathrm{I}_{\mathrm{K}}\right]^{-1}
$$

where $\sigma_{\mathrm{x}}{ }^{2}$ and $\sigma_{\mathrm{n}}{ }^{2}$ are the transmitted original signal and the received noise signal variances correspondingly. For a quantized massive MU-MIMO system, we have the Kth element of $\hat{x}$ as given by (8),

$$
\hat{\mathrm{x}}=\mathrm{A}^{\mathrm{H}} \mathrm{r}=\mathrm{A}^{\mathrm{H}} \mathcal{Q}(\mathrm{y})=\mathrm{A}^{\mathrm{H}} \mathcal{Q}(\mathrm{Hx}+\mathrm{n})=\mathrm{a}_{\mathrm{K}}{ }^{\mathrm{H}} \mathrm{h}_{\mathrm{K}} \mathrm{x}_{\mathrm{K}}+\sum_{\mathrm{i} \neq \mathrm{K}}^{\mathrm{K}} \mathrm{a}_{\mathrm{K}}{ }^{\mathrm{H}} \mathrm{h}_{\mathrm{i}} \mathrm{x}_{\mathrm{i}}+\mathrm{a}_{\mathrm{K}}{ }^{\mathrm{H}} \mathrm{n}
$$

where the first term in (8) represents the desired signal, the second term represents the interuser interference, and the third term represents the noise.

\section{SIMULATION RESULTS}

In this section of the paper, MATLAB simulations are applied to explain the effects of different quantization resolution levels of analog-to-digital converter (ADCs) on the BER performance in massive multiple input multiple output (MIMO) systems under different operating scenarios.

\subsection{BER performance using different schemes of modulation}

Figures 3 and 4 show the BER performances using two different schemes of quadrature amplitude modulation, 4 QAM and 16 QAM, and different quantization resolution levels of ADCs and LMMSE signal symbols detector have been used at the BS. Simulations results are implemented with 80 antennas at the transmitter and serving 5 antennas as a user's. The effects of different quantization resolution levels of ADCs on the BER performance are shown as a signal to noise ratio (SNR) get bigger, the result demonstrate that the BER degradation exponentially in a similar way for both schemes of modulations when using the same quantization resolution levels of ADCs. Nevertheless, the results illustrate that by increasing the quantization resolution levels of ADCs, the BER performance becomes more ideal.

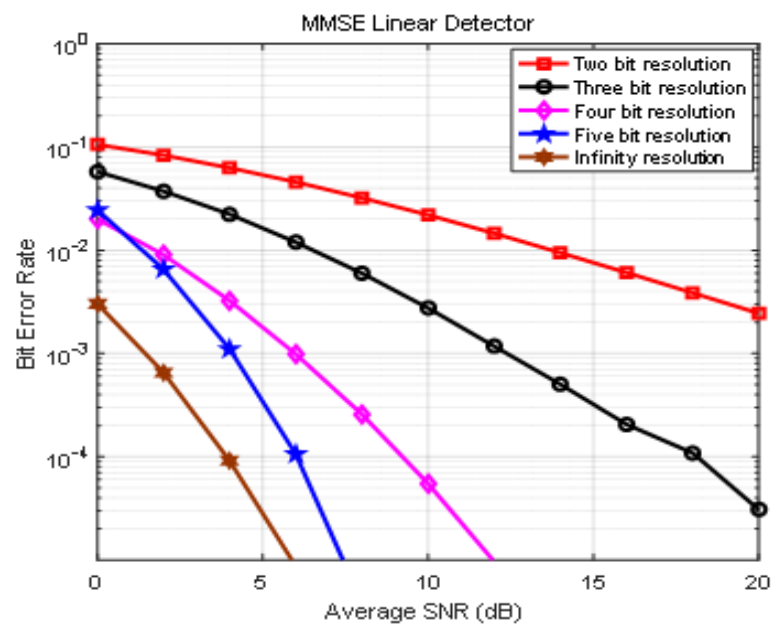

Figure 3. BER performance using different quantization resolution levels of ADCs, $T_{X}=80, R_{X}=5$, and 4 QAM

In order to study higher-order modulations, we observe that with using 2 and 3 bits-quantization resolution levels of ADCs, the BER showing degrades, while using the condition 4 bits-quantization resolution levels, the BER becomes acceptable. Furthermore, having 5 bits-quantization resolution levels and 
overhead, the BER effectiveness is very close to the infinity bits-quantization resolution levels. The outcomes show when using higher order of modulation (4 QAM and 16 QAM), the BER increases correspondingly for specific quantization resolution levels of ADCs. Consequently, higher quantization resolution levels of ADCs are obligatory for advanced order modulations to attain the equivalent performance.

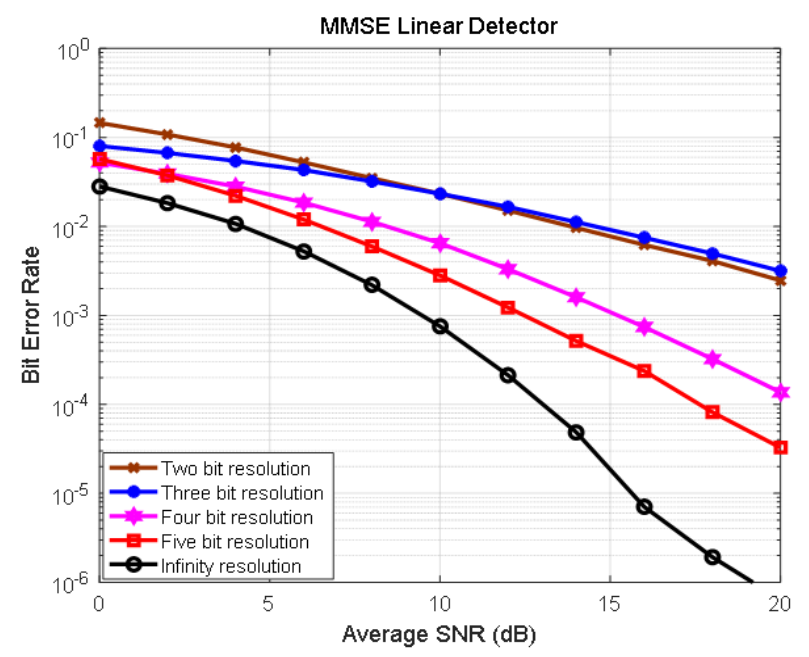

Figure 4. BER performance using different quantization resolution levels of $\mathrm{ADCs}, T_{X}=80, R_{X}=5$, and $16 \mathrm{QAM}$

\subsection{BER performance using different number of antennas}

Further simulation is accomplished to determine BER performance as a function of quantization resolution levels of ADCs with a different number of BS antennas, and LMMSE signal symbols detector have been used. We examine a constant number of users as a receiver $T_{X}=5$, and the number of antennas at the BS will be taken from 60 to 300 antennas. Figures 5 and 6 explain that increasing the number of antennas at the transmitter will decrease the BER regression caused by the low quantization resolution levels of ADCs. Figure 5 illustrate a 2 bits-quantization resolution levels ADCs with three different number of the antenna $(60,120$, and 300) has an obvious change with the increase the number of antennas. Comparably, with Figure 6, the BER performance of 4 bits-quantization resolution levels ADCs with the same different antennas have a slight degradation. Wherefore, the higher number of antennas at the BS is proposed to recover the BER performance regression in the situation of having constant low quantization resolution levels ADCs.

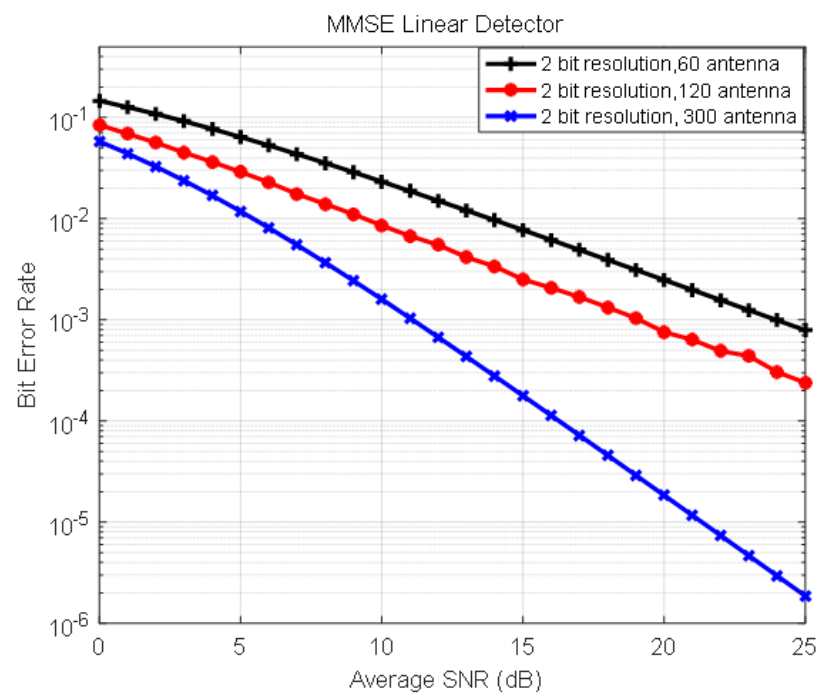

Figure 5. BER performance using 2 bit-quantization resolution levels ADCs and 4 QAM 


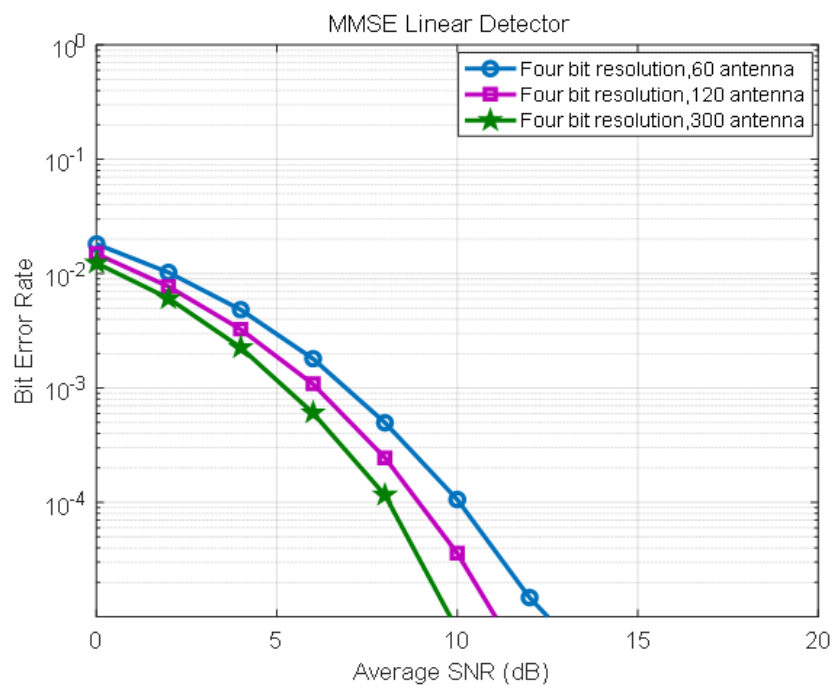

Figure 6. BER performance using 4 bit-quantization resolution levels ADCs and 4 QAM

\section{CONCLUSION}

In this work, we evaluated and measured the effects of different quantization resolution levels of ADCs on the BER performance of massive MIMO systems under different operating scenarios. The two typical modulations, 4 QAM and 16 QAM have been used with LMMSE signal symbols detector to evaluate the system. The results demonstrated that the performance of BER in massive MIMO technology is affected directly by the quantization resolution levels of ADCs, the number of antennas, and modulation schemes. Low (1 bit and 2 bits)-quantization resolution levels ADCs can realize appropriate BER performances compared to the high quantization resolution levels ADCs when the number of antennas at the BS high. Moreover, we observe that using 4 bits-quantization resolution levels ADCs in 16 QAM, the SNR of around $15.5 \mathrm{~dB}$ is obligatory to attained the BER of $10^{-3}$, whereas using 4 bits-quantization resolution levels ADCs in 4 QAM require only $6 \mathrm{~dB}$ SNR. We conclude that increasing the modulation order will significantly affect the BER performance of high-quantization resolution levels ADCs while slightly affected the BER performance when low-quantization resolution levels have been used.

\section{REFERENCES}

[1] Y. M. Tabra and B. M. Sabbar, "Hybrid MVDR-LMS Beamforming for Massive MIMO," Indonesian Journal of Electrical Engineering and Computer Science (IJEECS), vol. 16, no. 2, pp. 715-723, 2019, doi: 10.11591/ijeecs.v16.i2.pp715-723.

[2] P. Sunita, Rosalin Samantaray, Pradyumna Ku. Mohapatra, R. N. Panda and Padma Sahu, "A New Complexity Reduction Methods of V-BLAST MIMO System in a Communication Channel," International Journal of Informatics and Communication Technology (IJ-ICT), vol. 8, no. 1, pp. 29-38, 2019, doi: 10.11591/ijict.v8i1.pp29-38.

[3] O. Agboje, Nsikan Nkordeh, Uzairue Stanley Idiake, Ololade Oladoyin, Kennedy Okokpujie and Ibinabo BobManuel, "MIMO Channels: Optimizing Throughput and Reducing Outage by Increasing Multiplexing Gain," TELKOMNIKA Telecommunication, Computing, Electronics and Control, vol. 18, no. 1, pp. 419-426, 2020, doi: 10.12928/telkomnika.v18i1.8720.

[4] M. K. Hussein and N. N. Khamiss, "Integrating Millimeter Wave with Hybrid Precoding Multiuser Massive MIMO for 5G Communication," TELKOMNIKA Telecommunication, Computing, Electronics and Control, vol. 18, no. 1, pp. 90-98, 2020, doi: 10.12928/telkomnika.v18i1.13674.

[5] A. Nalband, M. Sarvagya, and M. R. Ahmed, "Power Saving and Optimal Hybrid Precoding in Millimeter Wave Massive MIMO Systems for 5G." TELKOMNIKA Telecommunication, Computing, Electronics and Control, vol. 18, no. 6, pp. 2842-2851, 2020, doi: 10.12928/telkomnika.v18i6.15952.

[6] L. Van der Perre, L. Liu, and E. G. Larsson, "Efficient DSP and Circuit Architectures for Massive MIMO: State of the Art and Future Directions," IEEE Transactions on Signal Processing, vol. 66, no. 18, pp. 4717-4736, Sept. 2018, doi: 10.1109/TSP.2018.2858190.

[7] M. A. Albreem, M. Juntti and S. Shahabuddin, "Massive MIMO Detection Techniques: A Survey," IEEE Communications Surveys \& Tutorials, vol. 21, no. 4, pp. 3109-3132, 2019, doi: 10.1109/COMST.2019.2935810.

[8] C. Stergiou, E. P. Kostas, B. B. Gupta and Y. Ishibashi, "Security, Privacy \& Efficiency of Sustainable Cloud Computing for Big Data \& IoT," Sustainable Computing: Informatics and Systems, vol. 19, pp. 174-184, 2018, doi: 10.1016/j.suscom.2018.06.003. 
[9] R. Yang, Shuqi Xi, Qibo Cai, Zhizhou Chen, Xiaohang Wang, Gui Liu, "A Compact Planar Dual-Band MultipleInput and Multiple-Output Antenna with High Isolation for 5G and 4G Applications," Micromachines, vol. 12, no. 5, pp. 1-8, May 2021, doi: 10.3390/mi12050544.

[10] P. Varzakas, "Average Channel Capacity for Rayleigh Fading Spread Spectrum MIMO Systems," International Journal of Communication Systems, vol. 19, no. 10, pp. 1081-1087, Dec. 2006, doi: 10.1002/dac.784.

[11] C. Wang, C. Wen, S. Jin, and S. Tsai, "Finite-Alphabet Precoding for Massive MU-MIMO with Low-Resolution DACs," IEEE Transactions on Wireless Communications, vol. 17, no. 7, pp. 4706-4720, July 2018, doi: 10.1109/twc.2018.2830343.

[12] S. Jacobsson, Giuseppe Durisi, Mikael Coldrey, Ulf Gustavsson, Christoph Studer, "Throughput Analysis of Massive MIMO Uplink with Low-Resolution ADCs," IEEE Transactions on Wireless Communications, vol. 16, no. 6, pp. 4038-4051, June 2017, doi: 10.1109/TWC.2017.2691318.

[13] J. Choi, Y. Cho, and B. L. Evans, "Quantized Massive MIMO Systems with Multicell Coordinated Beamforming and Power Control," IEEE Transactions on Communications, vol. 69, no. 2, pp. 946-961, Feb. 2021, doi: 10.1109/TCOMM.2020.3036689.

[14] T. Liu, Jun Tong, Qinghua Guo, Jiangtao Xi, Yanguang Yu and Zhitao Xiao, "Energy Efficiency of Massive MIMO Systems with Low-Resolution ADCs and Successive Interference Cancellation," IEEE Transactions on Wireless Communications, vol. 18, no. 8, pp. 3987-4002, Aug. 2019, doi: 10.1109/TWC.2019.2920129.

[15] J. Chen, S. Chen, Y. Qi, and S. Fu, "Intelligent Massive MIMO Antenna Selection Using Monte Carlo Tree Search," IEEE Transactions on Signal Processing, vol. 67, no. 20, pp. 5380-5390, Oct. 2019, doi: 10.1109/TSP.2019.2940128.

[16] Z. Xiao, Jincan Zhao, Tianle Liu, Lei Geng, F. Zhang and Jun Tong, "On the Energy Efficiency of Massive MIMO Systems with Low-Resolution ADCs and Lattice Reduction Aided Detectors," Symmetry, vol. 12, no.3, pp. 1-20, 2020, doi: 10.3390/sym12030406.

[17] H. Wang, W. Shih, C. Wen, and S. Jin, "Reliable OFDM Receiver with Ultra-Low Resolution ADC," IEEE Transactions on Communications, vol. 67, no. 5, pp. 3566-3579, May 2019, doi: 10.1109/TCOMM.2019.2894629.

[18] J. Liu, Z. Luo, and X. Xiong, "Low-Resolution ADCs for Wireless Communication: A Comprehensive Survey," in IEEE Access, vol. 7, pp. 91291-91324, 2019, doi: 10.1109/ACCESS.2019.2927891.

[19] J. Zhang, Linglong Dai, Ziyan He, Shi Jin, and Xu Li, "Performance Analysis of Mixed-ADC Massive MIMO Systems Over Rician Fading Channels," IEEE Journal on Selected Areas in Communications, vol. 35, no. 6, pp. 1327-1338, 2017, doi: 10.1109/JSAC.2017.2687278.

[20] T. Wadayama and S. Takabe, "Quantizer Optimization Based on Neural Quantizerfor Sum-Product Decoder," in 2018 IEEE Global Communications Conference (GLOBECOM), Abu Dhabi, United Arab Emirates, 2018, pp. 1-6, doi: 10.1109/GLOCOM.2018.8647503.

[21] Z. Shao, R. C. de Lamare, and L. T. N. Landau, "Iterative Detection and Decoding for Large-Scale MultipleAntenna Systems with 1-Bit ADCs," IEEE Wireless Communications Letters, vol. 7, no. 3, pp. 476-479, June 2018, doi: 10.1109/LWC.2017.2787159.

[22] C. Chen, W. Zhang, and X. Bao, "Achievable Rate Analysis on the Uplink of Massive MIMO with Superimposed Pilots and Arbitrary-Bit ADCs," in 2019 IEEE 19th International Conference on Communication Technology (ICCT), Xi'an, China, 2019, pp. 674-678, doi: 10.1109/ICCT46805.2019.8947297.

[23] Y. Dong and L. Qiu, "Spectral Efficiency of Massive MIMO Systems with Low-Resolution ADCs and MMSE Receiver," IEEE Communications Letters, vol. 21, no. 8, pp. 1771-1774, Aug. 2017, doi: 10.1109/LCOMM.2017.2693276.

[24] R. Bhandari and S. Jadhav, "Spectral Efficient Blind Channel Estimation Technique for MIMO-OFDM Communications," International Journal of Advances in Applied Sciences (IJAAS), vol. 7, no. 3, pp. 286-297, 2018, doi: 10.11591/ijaas.v7.i3.pp286-297.

[25] Ö. T. Demir and E. Björnson, "ADMM-Based One-Bit Quantized Signal Detection for Massive MIMO Systems with Hardware Impairments," in ICASSP 2020-2020 IEEE International Conference on Acoustics, Speech and Signal Processing (ICASSP), Barcelona, Spain, 2020, pp. 9120-9124, doi: 10.1109/icassp40776.2020.9053984.

[26] S. Jacobsson, Giuseppe Durisi, Mikael Coldrey, Ulf Gustavsson and Christoph Studer, "One-Bit Massive MIMO: Channel Estimation and High-Order Modulations," in 2015 IEEE International Conference on Communication Workshop (ICCW), London, UK, 2015, pp. 1304-1309, doi: 10.1109/ICCW.2015.7247358.

[27] V. Bhatia, M. R. Tripathy, and P. Ranjan, "Deep Learning for Massive MIMO: Challenges and Future Prospects," in 2020 IEEE 9th International Conference on Communication Systems and Network Technologies (CSNT), Gwalior, India, pp. 26-31, 2020, doi: 10.1109/CSNT48778.2020.9115783.

[28] L. V. Nguyen, Duy Trong Ngo, Nghi H. Tran, A. Lee Swindlehurst and Duy H. N. Nguyen, "Supervised and SemiSupervised Learning for MIMO Blind Detection with Low-Resolution ADCs," IEEE Transactions on Wireless Communications, vol. 19, no. 4, pp. 2427-2442, April 2020, doi: 10.1109/twc.2020.2964661. 